\title{
Existence of Weak Solutions for a Nonlocal Problem Involving the $p(x)$-Laplace Operator
}

\author{
Mustafa Avci \\ Faculty of Economics and Administrative Sciences, Batman University, 72000 Batman, Turkey \\ ${ }^{*}$ Corresponding Author: avcixmustafa@gmail.com
}

Copyright (C)2013 Horizon Research Publishing All rights reserved.

\begin{abstract}
This paper deals with the existence of weak solutions for some nonlocal problem involving the $p(x)$ Laplace operator. Using a direct variational method and the theory of the variable exponent Sobolev spaces, we set some conditions that ensures the existence of nontrivial weak solutions.
\end{abstract}

Keywords $p(x)$-Laplace operator, $p(x)$-Kirchhoff-type equations, variable exponent Sobolev spaces, variational method, mountain pass theorem, Ekeland variational principle

MSC: 35D05; 35J60; 35J70

\section{Introduction}

In the present paper, we are concerned with the Dirichlet boundary value problem

$$
\left\{\begin{array}{c}
-M\left(\int_{\Omega} \frac{|\nabla u|^{p(x)}}{p(x)} d x\right) \Delta_{p(x)} u=\lambda a(x)|u|^{q(x)-2} u \text { in } \Omega, \\
u=0 \quad \text { on } \partial \Omega,
\end{array}\right.
$$

where $\Omega \subset \mathbb{R}^{N}, N \geq 3$, is a smooth bounded domain, $\lambda>0 ; p, q \in C(\bar{\Omega})$ and $a$ is a non-negative measurable real-valued function, $M$ is a continuous function which obey some specific conditions. The purpose of the present paper is to find a nontrivial weak solution for a $p(x)$-Kirchhoff-type equation $(\mathbf{P})$ in the variable exponent Sobolev spaces. The main tool is variational approach. By help of the well-known theorems mountain pass theorem and Ekeland variational principle, the existence result is obtained.

Problem $(\mathbf{P})$ is related to the stationary version of a model, the so-called Kirchhoff equation, introduced by Kirchhoff [14]. To be more precise, Kirchhoff established a model given by the equation

$$
\rho \frac{\partial^{2} u}{\partial t^{2}}-\left(\frac{P_{0}}{h}+\frac{E}{2 L} \int_{0}^{L}\left|\frac{\partial u}{\partial x}\right|^{2} d x\right) \frac{\partial^{2} u}{\partial x^{2}}=0
$$

where $\rho, P_{0}, h, E, L$ are constants, which extends the classical D'Alambert's wave equation, by considering the effects of the changes in the length of the strings during the vibrations. For $p(x)$-Kirchhoff-type equations see, for example, $[4,6,12]$.

The importance of problem $(\mathbf{P})$ arises mainly from the existence of the $p(x)$-Laplacian $\triangle_{p(x)} u=\operatorname{div}\left(|\nabla u|^{p(x)-2} \nabla u\right)$. Obviously, when $p(x)=2, \triangle_{2}=\triangle$ is the usual Laplace operator. However, in case $p(x) \neq 2$ the situation is very crucial, as for example, one encounters the lack of the homogeneity, and a result of this, some classical theories, such as the theory of Sobolev spaces, is not applicable. For the papers involving the $p(x)$-Laplacian operator we refer the readers to $[3,5,11,13,16,17,18]$ and references therein. Moreover, the nonlinear problems involving the $p(x)$-Laplacian extremely attractive because they can be used to model dynamical phenomena which arise from the study of electrorheological fluids or elastic mechanics. Problems with variable exponent growth conditions also appear in the modelling of stationary thermo-rheological viscous flows of non-Newtonian fluids and in the mathematical description of the processes filtration of an ideal barotropic gas through a porous medium. The detailed application backgrounds of the $p(x)$-Laplacian can be found in $[1,2,19,20,22]$ and references therein. 


\section{Preliminaries}

We state some basic properties of the variable exponent Lebesgue-Sobolev spaces $L^{p(x)}(\Omega)$ and $W^{1, p(x)}(\Omega)$, where $\Omega \subset \mathbb{R}^{N}$ is a bounded domain.In that context we refer to $[7,9,10,15]$ for the fundamental properties of these spaces.

Set

$$
C_{+}(\bar{\Omega})=\{p: p \in C(\bar{\Omega}), p(x)>1 \text { for any } x \in \bar{\Omega}\} .
$$

For any $p \in C_{+}(\bar{\Omega})$, denote $1<p^{-}:=\inf _{x \in \bar{\Omega}} p(x) \leq p(x) \leq p^{+}:=\sup _{x \in \bar{\Omega}} p(x)<\infty$, and define the variable exponent Lebesgue space by

$$
L^{p(x)}(\Omega)=\left\{u \mid u: \Omega \rightarrow \mathbb{R} \text { is measurable, } \int_{\Omega}|u(x)|^{p(x)} d x<\infty\right\} .
$$

We define a norm, the so-called Luxemburg norm, on this space by the formula

$$
|u|_{p(x)}=\inf \left\{\eta>0: \int_{\Omega}\left|\frac{u(x)}{\eta}\right|^{p(x)} d x \leq 1\right\},
$$

and $\left(L^{p(x)}(\Omega),|\cdot|_{p(x)}\right)$ becomes a Banach space.

The conjugate space of $L^{p(x)}(\Omega)$ is $L^{p^{\prime}(x)}(\Omega)$, where $\frac{1}{p^{\prime}(x)}+\frac{1}{p(x)}=1$. For any $u \in L^{p(x)}(\Omega)$ and $v \in L^{p^{\prime}(x)}(\Omega)$, we have

$$
\left|\int_{\Omega} u v d x\right| \leq\left(\frac{1}{p^{-}}+\frac{1}{\left(p^{-}\right)^{\prime}}\right)|u|_{p(x)}|v|_{p^{\prime}(x)},
$$

which is known as Hölder inequality. The modular of $L^{p(x)}(\Omega)$ is $\rho_{p(x)}: L^{p(x)}(\Omega) \rightarrow \mathbb{R}$ defined by

$$
\rho_{p(x)}(u)=\int_{\Omega}|u(x)|^{p(x)} d x .
$$

If $u, u_{n} \in L^{p(x)}(\Omega), n=1,2, \ldots$, and $p^{+}<\infty$, we have

$$
\begin{aligned}
(i)|u|_{p(x)} & >1 \Longrightarrow|u|_{p(x)}^{p^{-}} \leq \rho_{p(x)}(u) \leq|u|_{p(x)}^{p^{+}} ; \\
(i i)|u|_{p(x)} & <1 \Longrightarrow|u|_{p(x)}^{p^{+}} \leq \rho_{p(x)}(u) \leq|u|_{p(x)}^{p^{-}} ; \\
(i i i)\left|u_{n}-u\right|_{p(x)} & \rightarrow 0 \Longleftrightarrow \rho_{p(x)}\left(u_{n}-u\right) \rightarrow 0 .
\end{aligned}
$$

We also consider the weighted variable exponent Lebesgue spaces. Let $b: \Omega \rightarrow \mathbb{R}$ is a measurable real function such that $b(x)>0$ a.e. $x \in \Omega$. We define

$$
L_{b(x)}^{p(x)}(\Omega)=\left\{u \mid u: \Omega \rightarrow \mathbb{R} \text { is measurable, } \int_{\Omega} b(x)|u(x)|^{p(x)} d x<\infty\right\} .
$$

The space $L_{b(x)}^{p(x)}(\Omega)$ endowed with the above norm is a Banach space which has similar properties with the variable exponent Lebesgue spaces. The modular of $L_{b(x)}^{p(x)}(\Omega)$ is $\rho_{(p(x), b(x))}: L_{b(x)}^{p(x)}(\Omega) \rightarrow \mathbb{R}$ defined by

$$
\rho_{(p(x), b(x))}(u)=\int_{\Omega} b(x)|u(x)|^{p(x)} d x .
$$

If $u, u_{n} \in L_{b(x)}^{p(x)}(\Omega)(n=1,2, \ldots)$ and $p^{+}<\infty$, we have

$$
\begin{aligned}
(i)\left|u_{n}\right|_{(p(x), b(x))} & >1 \Longrightarrow|u|_{(p(x), b(x))}^{p^{-}} \leq \rho_{p(x)}(u) \leq|u|_{(p(x), b(x))}^{p^{+}} ; \\
(i i)\left|u_{n}\right|_{(p(x), b(x))} & <1 \Longrightarrow|u|_{(p(x), b(x))}^{p^{+}} \leq \rho_{p(x)}(u) \leq|u|_{(p(x), b(x))}^{p^{-}} .
\end{aligned}
$$

The variable exponent Sobolev space $W^{1, p(x)}(\Omega)$ is defined by

$$
W^{1, p(x)}(\Omega)=\left\{u \in L^{p(x)}(\Omega) ; \quad|\nabla u| \in L^{p(x)}(\Omega)\right\},
$$

then it can be equipped with the norm

$$
\|u\|_{1, p(x)}=|u|_{p(x)}+|\nabla u|_{p(x)}, \forall u \in W^{1, p(x)}(\Omega) .
$$

The space $W_{0}^{1, p(x)}(\Omega)$ is denoted by the closure of $C_{0}^{\infty}(\Omega)$ in $W^{1, p(x)}(\Omega)$. We will use $\|u\|=|\nabla u|_{p(x)}$ for $u \in W_{0}^{1, p(x)}(\Omega)$ in the following discussions. 
Moreover, if $1<p^{-} \leq p^{+}<\infty$ the spaces $L^{p(x)}(\Omega), W^{1, p(x)}(\Omega)$ and $W_{0}^{1, p(x)}(\Omega)$ are separable and reflexive Banach spaces [15].

Proposition 2.2 $[9,15]$ Let $q \in C_{+}(\bar{\Omega})$. If $q(x)<p^{*}(x)$ for all $x \in \bar{\Omega}$, then the embedding $W^{1, p(x)}(\Omega) \hookrightarrow$ $L^{q(x)}(\Omega)$ is compact and continuous, where $p^{*}(x)=\frac{N p(x)}{N-p(x)}$ if $p(x)<N$ and $p^{*}(x)=+\infty$ if $p(x) \geq N$.

Proposition 2.3 [11] Let $X$ be a Banach space and $\Lambda(u)=\int_{\Omega} \frac{|\nabla u|^{p(x)}}{p(x)} d x$. The functional $\Lambda: X \rightarrow \mathbb{R}$ is convex. The mapping $\Lambda^{\prime}: X \rightarrow X^{*}$ is a strictly monotone, bounded homeomorphism, and of $\left(S_{+}\right)$type, namely

$$
u_{n} \rightarrow u \text { (weakly) and } \varlimsup_{n \rightarrow \infty}\left\langle\Lambda^{\prime}\left(u_{n}\right), u_{n}-u\right\rangle \leq 0 \text { implies } u_{n} \rightarrow u \text { (strongly). }
$$

\section{The Main Results}

We say that $u \in W_{0}^{1, p(x)}(\Omega)$ is a weak solution of $(\mathbf{P})$ if

$$
M\left(\int_{\Omega} \frac{|\nabla u|^{p(x)}}{p(x)} d x\right) \int_{\Omega}|\nabla u|^{p(x)-2} \nabla u \nabla \varphi d x-\lambda \int_{\Omega} a(x)|u|^{q(x)-2} u \varphi d x=0,
$$

where $\varphi \in W_{0}^{1, p(x)}(\Omega)$.

The energy functional corresponding to problem $(\mathbf{P})$ is defined as $J_{\lambda}: W_{0}^{1, p(x)}(\Omega) \rightarrow \mathbb{R}$,

$$
J_{\lambda}(u)=\widehat{M}\left(\int_{\Omega} \frac{|\nabla u|^{p(x)}}{p(x)} d x\right)-\lambda \int_{\Omega} a(x) \frac{|u|^{q(x)}}{q(x)} d x
$$

where $\widehat{M}(t)=\int_{0}^{t} M(s) d s$ for $t \in \mathbb{R}$. It is not difficult to show that $J_{\lambda} \in C^{1}\left(W_{0}^{1, p(x)}(\Omega), \mathbb{R}\right)$, and

$$
\left\langle J_{\lambda}^{\prime}(u), v\right\rangle=M\left(\int_{\Omega} \frac{|\nabla u|^{p(x)}}{p(x)} d x\right) \int_{\Omega}|\nabla u|^{p(x)-2} \nabla u \nabla v d x-\lambda \int_{\Omega} a(x)|u|^{q(x)-2} u v d x,
$$

for any $u, v \in W_{0}^{1, p(x)}(\Omega)$. Hence, we can infer that critical points of functional $J_{\lambda}$ are the weak solutions for problem $(\mathbf{P})$.

The main result of the present paper is:

Theorem 3.1. Suppose the following conditions hold:

$\left(M_{1}\right) M: \mathbb{R}^{+} \rightarrow \mathbb{R}^{+}$is a continuous function and satisfies the condition

$$
m_{1} t^{\alpha-1} \leq M(t) \leq m_{2} t^{\alpha-1}
$$

for all $t>0$, where $m_{1}, m_{2}$ and $\alpha$ are real numbers such that $0<m_{1} \leq m_{2}$ and $\alpha \geq 1$;

$\left(P_{1}\right) a \in L^{\delta(x)}(\Omega), a(x)>0$ and $\delta \in C_{+}(\bar{\Omega})$ such that $\frac{1}{\delta(x)}+\frac{1}{\delta_{0}(x)}=1, p(x)<\delta_{0}(x) q(x)$ and $1<q(x)<$ $\frac{1}{\delta_{0}(x)} p^{*}(x) \forall x \in \bar{\Omega}$;

$\left(P_{2}\right) 1<q^{-}<p^{-}<q^{+}, p^{+}<N$.

Then, there exists $\lambda^{*}>0$ such that $(\mathbf{P})$ has a nontrivial weak solution for any $\lambda \in\left(0, \lambda^{*}\right)$.

The proof of Theorem 3.1 based on the Mountain-Pass theorem [21] and the Ekeland's variational principle [8], and it is broken into several parts.

Lemma 3.2 Assume that $\left(M_{1}\right),\left(P_{1}\right)$ and $\left(P_{2}\right)$ hold. Then there exist positive real numbers $\gamma, r$ and $\lambda^{*}>0$ such that for any $\lambda \in\left(0, \lambda^{*}\right)$, we have $J_{\lambda}(u) \geq r>0, u \in W_{0}^{1, p(x)}(\Omega)$ with $\|u\|=\gamma$.

Proof. By using assumption $\left(P_{1}\right)$, and the arguments developed in [16, Theorem 2.8], we can write

$$
\int_{\Omega} a(x)|u|^{q(x)} d x \leq C\left(\|u\|^{q^{-}}+\|u\|^{q^{+}}\right) .
$$

Consider $\gamma \in(0,1)$. Then the above relation implies

$$
\int_{\Omega} a(x)|u|^{q(x)} d x \leq C\|u\|^{q^{-}}, \forall u \in W_{0}^{1, p(x)}(\Omega) .
$$

Using $\left(M_{1}\right),\left(P_{1}\right),(1.2)$ and $(3.2)$, we obtain that for any $u \in W_{0}^{1, p(x)}(\Omega)$ with $\|u\|=\gamma$ the following inequalities hold true 


$$
\begin{aligned}
& J_{\lambda}(u)=\widehat{M}\left(\int_{\Omega} \frac{|\nabla u|^{p(x)}}{p(x)} d x\right)-\lambda \int_{\Omega} a(x) \frac{|u|^{q(x)}}{q(x)} d x \\
& \geq \int_{0}^{\frac{1}{p^{+} \rho_{p(x)}(\nabla u)}} s^{\alpha-1} d s-\frac{\lambda}{q^{-}} \int_{\Omega} a(x)|u|^{q(x)} d x \\
& \geq \frac{m_{1}}{\alpha\left(p^{+}\right)^{\alpha}}\left(\int_{\Omega}|\nabla u|^{p(x)} d x\right)^{\alpha}-\frac{\lambda}{q^{-}} \int_{\Omega} a(x)|u|^{q(x)} d x \\
& \geq \frac{m_{1}}{\alpha\left(p^{+}\right)^{\alpha}}\|u\|^{\alpha p^{+}}-\frac{\lambda C}{q^{-}}\|u\|^{q^{-}} \\
& =\left(\frac{m_{1}}{\alpha\left(p^{+}\right)^{\alpha}} \gamma^{\alpha p^{+}-q^{-}}-\frac{\lambda C}{q^{-}}\right) \gamma^{q^{-}} .
\end{aligned}
$$

In the last inequality above, if we choose

$$
\lambda^{*}=\frac{m_{1} q^{-}}{\alpha\left(p^{+}\right)^{\alpha} C} \gamma^{\alpha p^{+}-q^{-}},
$$

then it is clear that there exists $r>0$ such that for any $\lambda \in\left(0, \lambda^{*}\right)$ we have

$$
J_{\lambda}(u) \geq r, \forall u \in W_{0}^{1, p(x)}(\Omega) \text { with }\|u\|=\gamma .
$$

The proof is complete.

Lemma 3.3 Assume that $\left(P_{1}\right)$ and $\left(P_{2}\right)$ hold. Then there exists $\varphi \in W_{0}^{1, p(x)}(\Omega)$ such that $\varphi \geq 0, \varphi \neq 0$ and $J_{\lambda}(t \varphi)<0$ for $t>0$ small enough.

Proof. From assumption $\left(P_{2}\right)$ we know that $q^{-}<p^{-}$. Let $\epsilon_{0}>0$ be such that $q^{-}+\epsilon_{0}<p^{-}$. On the other hand, since $q \in C(\bar{\Omega})$ it follows that there exists an open set $\Omega_{0} \subset \Omega$ such that $\left|q(x)-q^{-}\right|<\epsilon_{0}$ for all $x \in \bar{\Omega}_{0}$. Thus, we conclude that $q(x) \leq q^{-}+\epsilon_{0}<p^{-}$for all $x \in \bar{\Omega}_{0}$.

Let $\varphi \in C_{0}^{\infty}(\Omega)$ be such that $\operatorname{supp}(\varphi) \supset \bar{\Omega}_{0}, \varphi(x)=1$ for all $x \in \bar{\Omega}_{0}$ and $0 \leq \varphi(x) \leq 1$ in $\Omega$. Then from the above facts for any $t \in(0,1)$ it follows

$$
\begin{aligned}
J_{\lambda}(t \varphi) & =\widehat{M}\left(\int_{\Omega} \frac{|\nabla t \varphi|^{p(x)}}{p(x)} d x\right)-\lambda \int_{\Omega} \frac{a(x)}{q(x)}|t \varphi|^{q(x)} d x \\
& \leq \frac{m_{2} t^{\alpha p^{-}}}{\alpha\left(p^{-}\right)^{\alpha}}\left(\int_{\Omega}|\nabla \varphi|^{p(x)} d x\right)^{\alpha}-\frac{\lambda}{q^{+}} \int_{\Omega} a(x)|t \varphi|^{q(x)} d x \\
& \leq \frac{m_{2} t^{\alpha p^{-}}}{\alpha\left(p^{-}\right)^{\alpha}}\left(\int_{\Omega}|\nabla \varphi|^{p(x)} d x\right)^{\alpha}-\frac{\lambda t^{q^{-}+\epsilon_{0}}}{q^{+}} \int_{\Omega_{0}} a(x)|\varphi|^{q(x)} d x .
\end{aligned}
$$

Therefore,

$$
J_{\lambda}(t \varphi)<0,
$$

for $0<t<\sigma^{1 / \alpha p^{-}-q^{-}-\epsilon_{0}}$ with

$$
0<\sigma<\min \left\{1, \frac{\lambda \alpha\left(p^{-}\right)^{\alpha}}{m_{2} q^{+}} \cdot \frac{\int_{\Omega_{0}} a(x)|\varphi|^{q(x)} d x}{\left(\int_{\Omega}|\nabla \varphi|^{p(x)} d x\right)^{\alpha}}\right\} .
$$

Finally, we remark that $\int_{\Omega}|\nabla \varphi|^{p(x)} d x>0$. Indeed, it is obvious that

$$
\int_{\Omega_{0}} a(x)|\varphi|^{q(x)} d x \leq \int_{\Omega} a(x)|\varphi|^{q(x)} d x \leq \int_{\Omega_{0}} a(x)|\varphi|^{q^{-}} d x .
$$

On the other hand, from (3.1) we know that there exists a positive constant $c$ such that

$$
\int_{\Omega_{0}} a(x)|\varphi|^{q^{-}} d x \leq c\|\varphi\| .
$$

From the above inequalities we get $\|\varphi\|>0$. Using the relations $(1.2)-(1.3)$, we deduce that $\int_{\Omega}|\nabla \varphi|^{p(x)} d x>0$. The proof is complete. 
Proof of Theorem 3.1. From Lemma 3.2, we infer that there exists a ball centered at the origin $\overline{B_{\rho}(0)} \subset$ $W_{0}^{1, p(x)}(\Omega)$, such that

$$
\inf _{\partial B_{\rho}(0)} J_{\lambda}>0
$$

Furthermore, by Lemma 3.3, we know that there exists $\varphi \in W_{0}^{1, p(x)}(\Omega)$ such that $J_{\lambda}(t \varphi)<0$ for $t>0$ small enough. Therefore, considering also inequality (3.3), we obtain that

$$
-\infty<c:=\inf _{B_{\rho}(0)} J_{\lambda}<0 .
$$

Let choose $\varepsilon>0$. Then, it follows

$$
0<\varepsilon \leq \inf _{\partial B_{\rho}(0)} J_{\lambda}-\inf _{B_{\rho}(0)} J_{\lambda}
$$

Now, if we apply the Ekeland's variational principle [8] to the functional $J_{\lambda}: \overline{B_{\rho}(0)} \rightarrow \mathbb{R}$, it follows that there exists $u_{\varepsilon} \in \overline{B_{\rho}(0)}$ such that

$$
\begin{aligned}
& J_{\lambda}\left(u_{\varepsilon}\right)<\inf _{B_{\rho}(0)} J_{\lambda}+\varepsilon, \\
& J_{\lambda}\left(u_{\varepsilon}\right)<J_{\lambda}(u)+\varepsilon\left\|u-u_{\varepsilon}\right\|, \quad u_{\varepsilon} \neq u .
\end{aligned}
$$

By the fact that

$$
J_{\lambda}\left(u_{\varepsilon}\right)<\inf _{B_{\rho}(0)} J_{\lambda}+\varepsilon<\inf _{B_{\rho}(0)} J_{\lambda}+\varepsilon<\inf _{\partial B_{\rho}(0)} J_{\lambda}
$$

we can infer that $u_{\varepsilon} \in B_{\rho}(0)$.

Now, let define $\Phi_{\lambda}: \overline{B_{\rho}(0)} \rightarrow \mathbb{R}$ by $\Phi_{\lambda}(u)=J_{\lambda}(u)+\varepsilon\left\|u-u_{\varepsilon}\right\|$. It is not difficult to see that $u_{\varepsilon}$ is a minimum point of $\Phi_{\lambda}$, and thus

$$
\frac{\Phi_{\lambda}\left(u_{\varepsilon}+t v\right)-\Phi_{\lambda}\left(u_{\varepsilon}\right)}{t} \geq 0,
$$

for $t>0$ small enough and any $v \in B_{1}(0)$. By the above expression, we have

$$
\frac{J_{\lambda}\left(u_{\varepsilon}+t v\right)-J_{\lambda}\left(u_{\varepsilon}\right)}{t}+\varepsilon\|v\| \geq 0
$$

Letting $t \rightarrow 0$, we have

$$
\left\langle J_{\lambda}^{\prime}\left(u_{\varepsilon}\right), v\right\rangle+\varepsilon\|v\|>0,
$$

and this implies that $\left\|J_{\lambda}^{\prime}\left(u_{\varepsilon}\right)\right\| \leq \varepsilon$. So, we deduce that there exists a sequence $\left\{u_{n}\right\} \subset B_{\rho}(0)$ such that

$$
J_{\lambda}\left(u_{n}\right) \rightarrow c=\inf _{B_{\rho}(0)} J_{\lambda}<0 \text { and } J_{\lambda}^{\prime}\left(u_{n}\right) \rightarrow 0 .
$$

Hence, we have that $\left\{u_{n}\right\}$ is bounded in $W_{0}^{1, p(x)}(\Omega)$. Thus, there exists $u \in W_{0}^{1, p(x)}(\Omega)$ such that, up to a subsequence, $\left\{u_{n}\right\}$ converges weakly to $u$ in $W_{0}^{1, p(x)}(\Omega)$ so $\left\langle J_{\lambda}^{\prime}\left(u_{n}\right), u_{n}-u\right\rangle \rightarrow 0$. Therefore, we write

$$
\begin{aligned}
& \left\langle J_{\lambda}^{\prime}\left(u_{n}\right), u_{n}-u\right\rangle \\
= & M\left(\int_{\Omega} \frac{\left|\nabla u_{n}\right|^{p(x)}}{p(x)} d x\right) \int_{\Omega}\left|\nabla u_{n}\right|^{p(x)-2} \nabla u_{n}\left(\nabla u_{n}-\nabla u\right) d x-\lambda \int_{\Omega} a(x)\left|u_{n}\right|^{q(x)-2} u_{n}\left(u_{n}-u\right) d x \rightarrow 0 .
\end{aligned}
$$

Using $\left(P_{1}\right),(1.1)$ and Proposition 2.2, we get the compact embedding $W_{0}^{1, p(x)}(\Omega) \hookrightarrow L_{a(x)}^{q(x)}(\Omega)$ (see $[16$, Theorem 2.8]). Then, we obtain that

$$
\int_{\Omega} a(x)\left|u_{n}\right|^{q(x)-2} u_{n}\left(u_{n}-u\right) d x \rightarrow 0
$$

and hence, we obtain

$$
M\left(\int_{\Omega} \frac{\left|\nabla u_{n}\right|^{p(x)}}{p(x)} d x\right) \int_{\Omega}\left|\nabla u_{n}\right|^{p(x)-2} \nabla u_{n}\left(\nabla u_{n}-\nabla u\right) d x \rightarrow 0 .
$$

From $\left(M_{1}\right)$, we conclude that

$$
\int_{\Omega}\left|\nabla u_{n}\right|^{p(x)-2} \nabla u_{n}\left(\nabla u_{n}-\nabla u\right) d x \rightarrow 0
$$

Therefore, by Proposition 2.3, we get $u_{n} \rightarrow u$ (strongly) in $W_{0}^{1, p(x)}(\Omega)$, so we conclude that $u$ is a nontrivial weak solution for problem $(\mathbf{P})[21]$. The proof of Theorem 3.1 is complete. 


\section{REFERENCES}

[1] S. N. Antontsev and S. I. Shmarev, A model porous medium equation with variable exponent of nonlinearity: existence, uniqueness and localization properties of solutions, Nonlin. Anal. 60 (2005), 515-545.

[2] M. Avci, Ni-Serrin type equations arising from capillarity phenomena with nonstandard growth, Boundary Value Problems, 2013:55, (2013), no.1, 1-18.

[3] M. Avci, Existence and multiplicity of solutions for Dirichlet problems involving the $p(x)$-Laplace operator, Electronic Journal of Differential Equations, Vol. (2013) (14), pp. 1-9, (2013).

[4] M. Avci , B. Cekic and R.A. Mashiyev , Existence and multiplicity of the solutions of the $p(x)$-Kirchhoff type equation via genus theory, Math. Methods in the Applied Sciences 34 (2011), no.14, 1751-1759.

[5] B. Cekic and R.A. Mashiyev, Existence and localization results for $p(x)$-Laplacian via topological methods, Fixed Point Theory Appl. 2010, Article ID 120646 (2010).

[6] G. Dai and D. Liu, Infinitely many positive solutions for a $p(x)$-Kirchhoff-type equation, J. Math. Anal. Appl. 359 (2009), no.2, 704-710.

[7] L. Diening, P. Harjulehto, P. Hasto and M. Ruzicka, Lebesgue and Sobolev Spaces with Variable Exponents, Lecture Notes in Mathematics Vol. 2017, DOI: 10.1007/9783-642-18363-8, 2011.

[8] I. Ekeland, On the variational principle, J. Math. Anal. Appl. 47 (1974), 324-353.

[9] X. L. Fan, J. S. Shen and D. Zhao, Sobolev embedding theorems for spaces $W^{k, p(\cdot)}(\Omega)$, J. Math. Anal. Appl. 262 (2001), 749-760.

[10] X. L. Fan and D. Zhao, On the spaces $L^{p(x)}$ and $W^{m, p(x)}$, J. Math. Anal. Appl. 263 (2001), 424-446.
[11] X.L. Fan and Q. H. Zhang, Existence of solutions for $p(x)$-Laplacian Dirichlet problems, Nonlinear Anal. 52 (2003), 1843-1852.

[12] X.L. Fan, On nonlocal $p(x)$-Laplacian Dirichlet problems, Nonlinear Anal. 72 (2010), 3314-3323.

[13] P. Hästö, The $p(x)$-Laplacian and applications, J. Anal. 15 (2007), 53-62 (Special proceedings volume).

[14] G. Kirchhoff, Mechanik, Teubner, Leipzig, 1883.

[15] O. Kovăčik, J. Răkosnik, On spaces $L^{p(x)}$ and $W^{1, p(x)}$, Czechoslovak Math. J. 41 (116) (1991), 592-618.

[16] R. A. Mashiyev, S. Ogras, Z. Yucedag, M. Avci, The Nehari manifold approach for Dirichlet problem involving the $p(x)$-Laplacian equation, J. Korean Math. Soc. 47 (2010), No. 4, 845-860.

[17] R. A. Mashiyev, Some Properties of variable Sobolev capacity, TaiwaneseJournal of Mathematics, 12 (2008), no.3, 671-679.

[18] M. Mihăilescu and V. Rădulescu, On a nonhomogeneous quasilinear eigenvalue problem in Sobolev spaces with variable exponent, Proceedings Amer. Math. Soc. 135 (9) (2007), 2929-2937.

[19] M. Mihăilescu and V. Rădulescu, A multiplicity result for a nonlinear degenerate problem arising in the theory of electrorheological fluids, Proc. R. Soc. A, 462 (2006), 26252641.

[20] M. Růžička, Electrorheological fluids: Modeling and Mathematical Theory, Lecture Notes in Mathematics, Springer-Verlag, Berlin, 2000.

[21] M. Willem, Minimax Theorems, Birkhauser, Basel, 1996.

[22] V.V. Zhikov, Averaging of functionals of the calculus of variations and elasticity theory, Math. USSR. Izv. 9 (1987), 33-66. 